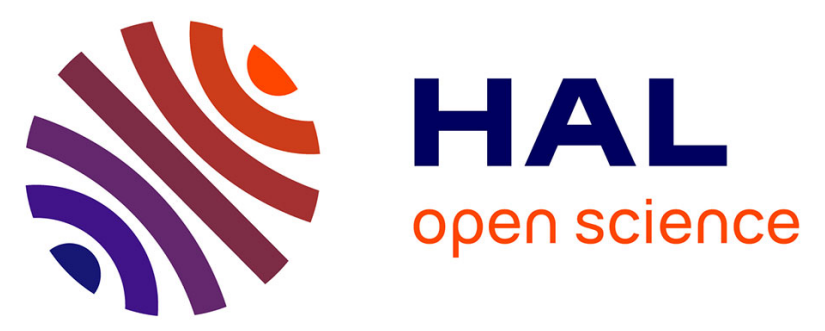

\title{
Influence of promised rewards on conflict resolution in healthy participants and patients with Parkinson's disease
}

Jean-François Houvenaghel, Joan Duprez, Florian Naudet, Soizic Argaud, Thibaut Dondaine, Sophie Drapier, Gabriel Hadrien Robert, Dominique Drapier, Marc Vérin, Paul Sauleau

\section{To cite this version:}

Jean-François Houvenaghel, Joan Duprez, Florian Naudet, Soizic Argaud, Thibaut Dondaine, et al.. Influence of promised rewards on conflict resolution in healthy participants and patients with Parkinson's disease. Journal of the Neurological Sciences, 2016, 367, pp.38-45. 10.1016/j.jns.2016.05.045 . hal-01324703

\section{HAL Id: hal-01324703 \\ https://hal-univ-rennes1.archives-ouvertes.fr/hal-01324703}

Submitted on 11 Oct 2016

HAL is a multi-disciplinary open access archive for the deposit and dissemination of scientific research documents, whether they are published or not. The documents may come from teaching and research institutions in France or abroad, or from public or private research centers.
L'archive ouverte pluridisciplinaire HAL, est destinée au dépôt et à la diffusion de documents scientifiques de niveau recherche, publiés ou non, émanant des établissements d'enseignement et de recherche français ou étrangers, des laboratoires publics ou privés. 


\section{Influence of promised rewards on conflict resolution in healthy participants and patients with Parkinson's disease}

Jean-François Houvenaghel ${ }^{\mathrm{a}, \mathrm{b}}$, Joan Duprez ${ }^{\mathrm{a}}$, Florian Naudet ${ }^{\mathrm{a}, \mathrm{c}, \mathrm{d}}$, Soizic Argaud ${ }^{\mathrm{a}, \mathrm{e}}$, Thibaut Dondaine $^{\mathrm{a}}$, Sophie Drapier ${ }^{\mathrm{a}, \mathrm{b}}$, Gabriel Hadrien Robert ${ }^{\mathrm{a}, \mathrm{d}}$, Dominique Drapier ${ }^{\mathrm{a}, \mathrm{d}}$, Marc Vérin ${ }^{\mathrm{a}, \mathrm{b}}$, Paul Sauleau ${ }^{\mathrm{a}, \mathrm{f}}$

a "Behavior and Basal Ganglia" research unit (EA 4712), University of Rennes 1, F-35033 Rennes, France

${ }^{\mathrm{b}}$ Department of Neurology, Rennes University Hospital, F-35033 Rennes, France

${ }^{\mathrm{c}}$ Clinical Investigation Center (INSERM 0203), Department of Pharmacology, Rennes University Hospital, F-35033 Rennes, France

${ }^{\mathrm{d}}$ Department of Adult Psychiatry, Rennes University Hospital, F-35033 Rennes, France

e "Neuroscience of Emotion and Affective Dynamics" laboratory, Department of Psychology and Educational Sciences, 40 boulevard du Pont d'Arve, 1205 Geneva, and Swiss Center for Affective Sciences, Campus Biotech, 1202 Geneva, University of Geneva, Switzerland

${ }^{\mathrm{f}}$ Department of Neurophysiology, Rennes University Hospital, F-35033 Rennes, France

Corresponding author: Jean-François Houvenaghel, Service de Neurologie, CHU Pontchaillou, 2 rue Henri Le Guilloux, 35033 Rennes Cedex, France. Phone: +(33)2 992898 42, Fax: +(33)2 992841 32, Email: jeanfrancois.houvenaghel@chu-rennes.fr

Word count: Abstract: 218 - Main text: 5893. 44 references, 2 tables, 4 figures; no supplemental data 


\begin{abstract}
The influence of promised rewards on conflict resolution processes is not clearly defined in the literature, and the underlying mechanisms are poorly understood. Some studies have shown no effect of reward, while others have demonstrated a beneficial influence. In addition, although the basal ganglia are known to play a critical role in the association between motivation and cognition, the influence of promised rewards on conflict resolution processes in Parkinson's disease (PD) has received little attention. In this context, we assessed the influence of promised rewards on both impulse activation and suppression in 36 healthy participants and 36 patients with PD, using a rewarded Simon task. Analysis of performances revealed that promised rewards worsened the overall congruence effect, but only in healthy participants. Although the incentive context did not modulate the congruence effect in patients, by using the activation-suppression model, we were able to show that promised rewards did influence impulse suppression in patients-but not in healthy participants. Suppressing inappropriate response activation in an incentive context appears to be harder in medically treated Parkinson's disease. This indicates that incentive motivation can modulate at least one cognitive process involved in cognitive action control in patients with medically treated PD. The activation-suppression model provides essential additional information concerning the influence of promised rewards on conflict resolution processes in a pathological population.
\end{abstract}

Keywords: motivation, reward, cognitive action control, Simon task, Parkinson's disease 


\section{Introduction}

Cognitive control is an important executive function commonly studied in contemporary neuropsychology in various neurological disorders, including Parkinson disease (PD) [1,2]. Cognitive action control refers to a subset of cognitive control processes that favor the production of goal-directed actions according to either internal objectives or environmental requirements. More specifically, cognitive action control allows for the expression of desired appropriate behavior, even if there is strong competition from an unintentional response triggered by irrelevant environmental information. Cognitive action control can be assessed in the laboratory with conflict tasks such as the Stroop task [3], the Eriksen task [4] or the Simon task [5]. The stimuli in the Stroop task and the Simon task usually carry both relevant and irrelevant features, which may activate either the same response or two different ones. In congruent situations, the relevant and irrelevant features of the stimulus both trigger the same response. By contrast, in noncongruent situations, the relevant and irrelevant features activate different responses, thereby creating conflict. Typically, compared with congruent situations, noncongruent ones induce slower and less accurate responses. The negative impact of conflict on response speed can be measured by the difference in reaction time for the correct responses in noncongruent minus congruent situations. This effect is known as the congruence effect. Dual-route models of information processing are now regarded as references for depicting the congruence effect in conflict tasks [6-8]. Different models have been proposed to conceptualize the cognitive control and have been used in various psychological domains [9]. However, dual-route models are considered to be more appropriate to describe the cognitive processes involved during online actions control, and especially during conflict resolution [10]. Dual-route models assume that the features of the stimuli trigger response tendencies via two distinct, parallel routes: an automatic, reflexive, route and a controlled one. The irrelevant feature of the stimulus activates a fast and automatic route, whereas the relevant 
feature activates a slower, controlled route. Furthermore, in conflict situations, selective response inhibition is required to suppress and overcome the irrelevant activation in favor of the relevant response. It is the cognitive cost of this process that is thought to induce the congruence effect.

According to the activation-suppression model [11-13], the selective inhibition of the irrelevant response tendency takes time to get going, and is therefore not immediately effective $[14,15]$. Accordingly, this model postulates that the efficiency of the top-down selective inhibition engaged to resolve conflict situations is greater for slow responses than for fast ones. Thus, in noncongruent situations, rapid responses are most vulnerable to inappropriate impulsive action selection captured by the irrelevant feature of the stimulus, resulting in fast, erroneous responses. Conversely, when responses are slower, selective inhibition has more time to build up and allows for the proper suppression of the automatic activation, facilitating the production of correct responses. Therefore, the congruence effect is lower for slow responses. Impulsive action selection can be revealed by the conditional accuracy function (CAF), which plots accuracy as a function of response speed. Impulsive selection is present when the fastest responses are the least accurate. Delta plots showing the relationship between the congruence effect and response speed, can be used to highlight response inhibition efficiency. Effective selective inhibition is observed when the congruence effect declines steeply for the slowest responses (for a review, see $[10,16]$ ).

According to motivation models in behavioral neurosciences, stimuli can motivated individuals by incentive expectancies (for review see [17]). The association of a hedonic reward to a neutral stimulus assigns an incentive value to the neutral stimulus. As a consequence, presentation of such a stimulus triggers expectation of the reward. Culturally, monetary coins have a powerful incentive value since they are strongly associated with 
hedonic processes. Accordingly, coins presentation may influence human behavior, including cognitive action control. However, relatively few studies have investigated the influence of promised rewards on the cognitive processes involved in the production of the desired action when a strong and undesired alternative is activated [18-21]. These studies have classically used rewarded conflict tasks, in which the promised rewards are displayed before the conflict situations (reward anticipation). A recent study featuring a rewarded Stroop-like task showed that the congruence effect was lower in rewarded than in nonrewarded situations, induced by response facilitation in both the congruent and noncongruent situations [20]. The authors therefore concluded that incentive motivation has a beneficial impact on cognitive action control. Other studies have failed to find any behavioral evidence of a significant influence of promised rewards on conflict resolution [18,21]. It should, however, be noted that these studies did not specifically investigate the influence of promised rewards on impulse selection and suppression. As a whole, therefore the influence of reward anticipation on cognitive action control is not yet fully understood.

Recent imaging studies in healthy participants have suggested that the basal ganglia and dopamine release could play a nodal role in the interaction between incentive motivation and cognitive action control [18-20]. In particular, Aarts et al. (2014) observed a relationship between dopamine synthesis capacity and behavioral performance in a motivated Stroop-like task [18]. Healthy participants with a high synthesis capacity in the left caudate nucleus exhibited a deleterious influence of promised rewards on cognitive action control (stronger congruence effect in high- vs. low-reward conditions). The authors suggested that in participants with a high dopamine synthesis capacity, the promise of a high rewards overdosed the dopamine system, leading to impaired cognitive action control. To date, there has only been one study of the influence of promised rewards on cognitive action control in 
conflict situations in PD, which represents a pathophysiological model of dysfunction in the basal ganglia and the dopamine system [19]. This study showed that rewarding the fastest responses-or punishing the slowest ones-in a Simon task had no effect on cognitive action control processes in patients with medically treated PD, contrary to controls. The authors suggested that dopaminergic treatment overdosed the ventral frontostriatal system which, in turn, prevented the incentive situation from modulating patients' cognitive action control.

In this context, the objective of the current study was to clarify the influence of promised rewards on the temporal aspects of information processing in conflict situations in both healthy participants and patients with medically treated PD. To this end, we used a Simon task in which incentive motivation was induced by monetary rewards.

\section{Methods}

\subsection{Participants.}

Two groups of 36 participants took part in the study: a group of healthy controls (HC) (age range: 44 - 71 years; education range: 6-17 years) with no neurological or psychiatric history, recruited by advertisement, and a group of patients with idiopathic PD [22] (age range: 29 71 years; education range: 6-20 years) undergoing medical care at Rennes University Hospital, France. Participants' clinical and demographic data are detailed in Table 1. Dementia was excluded using the Mattis Dementia Rating Scale (MDRS, [23]), and psychiatric disorders using the French version of the Mini-International Neuropsychiatric Interview (MINI 500, [24]). Trained psychiatrists assessed depressive symptoms on the Montgomery and Asberg Depressive Rating Scale (MADRS, [25]). PD severity was measured on the Unified Parkinson's Disease Rating Scale (UPDRS), and the Hoehn and Yahr [26] and Schwab and England [27] scales. All the participants, HC and patients, were examined in the department of Neurology of the Rennes University Hospital, France. Both groups were 
matched for age, sex, education level and MDRS score. Patients performed the experimental task on their usual antiparkinsonian medication in order to decrease the bias from motor impairment. Medication included levodopa and dopamine agonists in 28 patients, and levodopa only in 7 patients. The experiment was approved by the local ethics committee of Rennes University Hospital, and conducted in accordance with the Declaration of Helsinki and current French legislation (Huriet Act). 
Table 1: Clinical and demographic data (mean $\pm S D$ ) and comparisons between HC and PD groups using the Mann-Whitney $U$ test.

\begin{tabular}{|c|c|c|c|}
\hline & HC group & PD group & $p$ value \\
\hline Men/Women & $18 / 18$ & $18 / 18$ & \\
\hline Age (years) & $59.4 \pm 6.3$ & $56.6 \pm 9.2$ & 0.25 \\
\hline Education (years) & $12.1 \pm 3.8$ & $11.1 \pm 4.3$ & 0.26 \\
\hline Disease duration (years) & & $11.4 \pm 4.2$ & \\
\hline UPDRS-III "On" & & 8.0 & \\
\hline UPDRS-III "Off" & & $28.3 \pm 9.7$ & \\
\hline Schwab and England (\%) "On" & & $87.5 \pm 8.6$ & \\
\hline Schwab and England (\%) "Off" & & $71.2 \pm 16.7$ & \\
\hline Hoehn and Yahr "On" & & $0.9 \pm 0.7$ & \\
\hline Hoehn and Yahr "Off" & & $2.3 \pm 0.7$ & \\
\hline LEDD (mg) & & $1204 \pm 525$ & \\
\hline Dopa agonist (mg) & & $431 \pm 411$ & \\
\hline Levodopa (mg) & & $773 \pm 389$ & \\
\hline MADRS & $1.9 \pm 2.4$ & $4.8 \pm 5.1$ & 0.01 \\
\hline MDRS & $139.8 \pm 2.9$ & $139.4 \pm 3.9$ & 0.93 \\
\hline
\end{tabular}

\subsection{Materials and procedure.}

The stimulus presentation was programmed using E-prime Professional version 2.0 running on a DELL LATTITUDE E550 computer. The experimental task comprised a baseline phase (32 trials) and an experimental one (five blocks of 72 trials, with a short pause between each block). Each phase was preceded by a familiarization phase (12 trials before the baseline phase, and six trials before the first block of the experimental phase). The baseline phase 
consisted of a standard Simon task with no promised reward. The promised rewards were introduced in the experimental phase.

All the stimuli were displayed on a 23.6-inch black computer screen (Fig. 1). Each trial began with a cue displayed in the center of the screen for $1000 \mathrm{~ms}$. This cue was a white cross for the baseline phase, and a coin indicating the promised reward for each trial in the experimental phase. This coin was either $€ 1,1$ cent, or a chimeric combination of the two (i.e. no reward, as explicitly indicated to the participants). The cue was followed by a black screen for 700-1100 ms, after which a blue or yellow circle (target) appeared on the right or left side of the screen. Participants were asked to press a blue or yellow button on a computer keyboard, as quickly and accurately as possible, according to the color of the target and independently of its position. Thus, the position of the target defined the congruence of the trial (e.g. congruent: blue circle located on the side of the blue button; noncongruent: blue circle located on the side of the yellow button). The target disappeared as soon as participants pressed one of the two buttons. In the experimental phase, participants were instructed to press the button indicated by the color of the target as quickly and accurately as possible, in order to obtain the greatest reward, the size of the reward being proportional to the response speed for the correct responses. For correct responses, the size of the rewards gained by participants was actually calculated on a linear basis. To obtain the full promised reward, participants had to reduce their reaction time by at least one sixth of their mean reaction time (RT) recorded at baseline. For example, if their mean baseline RT was 480 ms, participants only obtained the full reward if they produced a correct response after no more than $400 \mathrm{~ms}$. A correct response with an RT equal to the baseline RT brought them $50 \%$ of the promised reward. Slower correct responses and incorrect responses were not rewarded. At the end of each trial, the amount of money won since the beginning of the experimental block was displayed for $1500 \mathrm{~ms}$, the maximum reward for each block being $€ 24.24$. The position of the 
color button was pseudorandomized across participants, and all the experimental conditions were pseudorandomized, with an equal number of trials in each condition. At baseline, each participant performed 16 congruent trials and 16 noncongruent ones. In the experimental phase, each combination of congruence (congruent/noncongruent) and level of reward (€1/1 cent/€0) was repeated 60 times per participant.
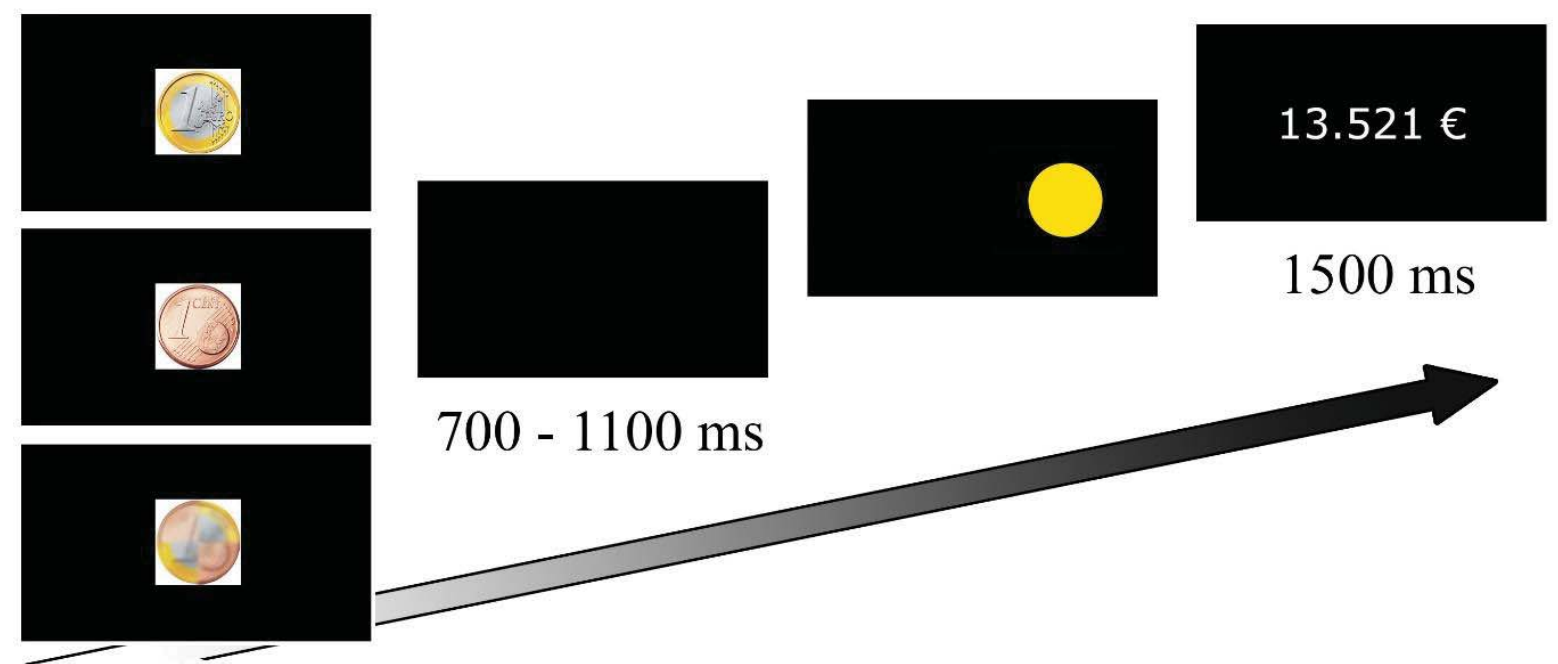

\section{$-1000 \mathrm{~ms}$}

Figure 1: At the beginning of each trial, the incentive monetary cue (€0, 1 cent or $€ 1)$ was displayed for 1000 ms. After an interval of 700-1100 ms, a blue or yellow circle appeared on the left or right side of the screen, and remained there until participants pressed the blue or yellow button (as quickly and accurately as possible). At the end of each trial, the size of the reward accumulated since the start of the experimental block was displayed for $1500 \mathrm{~ms}$.

\subsection{Statistical analysis.}

Statistical analyses were performed using R (version 3.1.0) implemented with the nlme [28] and lme4 [29] packages. Only responses from the experimental phase with an RT between $200 \mathrm{~ms}$ and $1500 \mathrm{~ms}$ and no more than three standard deviations below the mean were analyzed. These cut-off points removed $1.4 \%$ of the initial dataset. As RTs were not normally distributed, they were first converted using the logarithmic transformation. Statistical analyses 
were therefore based on response accuracy and the transformed RTs of correct responses. To avoid losing statistical power and to take interindividual variability into account, we chose to work on the whole dataset, and not with the averaged data per condition and per participant. A significance threshold of $p=0.05$ was used for all the statistical analyses.

We first analyzed the influence of the promised rewards on cognitive action control using a linear mixed model for RTs and a nonlinear mixed model for response accuracy. In each model, the fixed effects were group (2 levels: HC vs. PD), congruence (2 levels: congruent vs. noncongruent) and reward ( 3 levels: $€ 1,1$ cent and $€ 0$ ), and the random effect was participant. We then estimated the influence of the promised rewards on the temporal dynamics of cognitive action control. To assess impulsive action selection, we used the CAF, which plotted response accuracy as a function of RT for each condition and each participant, and divided each distribution into three equal parts (bins), depending on the RT. To analyze response accuracy, we used a nonlinear mixed model in which the fixed effects were group, congruence, reward and bin (3 levels: first, second and third bins), and the random effect was participant. To assess selective response suppression, we focused on changes in the congruence effect for correct responses according to RTs for each level of reward and each participant, and divided each distribution into three equal bins. We then estimated the slopes between these bins (delta plots, 2 slopes). To analyze these slopes (represented by $m$ in the Results section), we used a linear mixed model in which the fixed effects were group, reward and slope (2 levels: first slope and second slope) and the random effect was participant. The more negative the slope, the more effective the impulse suppression.

\section{Results}

This section is divided into three parts. The first part contains an analysis of the overall data, as classically reported in conflict task studies. In the second and third parts, we analyze the 
data according to the activation-suppression model: the second part is devoted to the CAF plots and the third to the delta plots. In each part, we first consider the effects of congruence and reward, and the interaction between the two, before proceeding to group comparisons. It should be noted that depression measured with the MADRS, which was the only unmatched variable between groups, had no effect on error rates, $F_{(1,25545)}=0.02, p=.88$, but did significantly influence RTs, $F_{(1,24478)}=3.77, p=.05$. The MADRS score was therefore added as a covariate in each statistical model to limit the influence of depression.

\subsection{Overall RTs and accuracy}

When we considered all the participants, we observed the classic congruence effect for RTs and for accuracy (Congruence, $\mathrm{RT}, F_{(1,24478)}=2956.81, p<.0001$; accuracy, $F_{(1,25545)}=$ 405.52, $p<.0001$ ) confirming that the selection of correct actions was harder in noncongruent condition than in congruent ones (Table 2). Irrespectively of congruence status, the promise of a reward increased the speed of correct responses (decreased RTs) (Reward, RT, $F_{(2,24478)}=$ 518.01, $p$.0001) but did not affect their accuracy (Reward, accuracy, $F_{(2,25545)}=2.79, p=$ .25). Responses were faster in the $€ 1$ situation (mean $\pm S D ; 487 \pm 122 \mathrm{~ms}$ ) than in either the 1 cent situation (Reward, RT- $€ 1$ vs. 1cent, $F_{(1,16344)}=159.49, p<.0001$ ), or the $€ 0$ situation (Reward, RT- $€ 1$ vs. $\left.€ 0, F_{(1,16296)}=511.86, p<.0001\right)$, and faster in the 1 cent situation than in the nonrewarded situation ( 1 cent: $504 \pm 124$ ms; $€ 0: 518 \pm 125$ ms) (Reward, RT- $€ 0$ vs. 1cent, $\left.F_{(1,16316)}=106.46, p<.0001\right)$. Furthermore, the promise of a reward modulated the congruence effects on both RTs (Congruence $\times$ Reward, RT, $F_{(2,24478)}=15.64, p=.0004$ ) and accuracy (Congruence $\times$ Reward, accuracy, $F_{(2,25545)}=7.28, p=.03$ ). Rewards increased response speeds in both congruent and noncongruent situations $(p<.0001)$, but only increased the error rate in noncongruent situations $(p=.04)$. In addition, the congruence effect was greater in the $€ 1$ situation than in the 1 cent situation for both RT (Congruence $\times$ Reward, 
RT $-€ 1$ vs. 1 cent, $\left.F_{(1,16344)}=5.15, p=.02\right)$ and accuracy (Congruence $\times$ Reward, errors1 cent vs. $\left.€ 1, F_{(1,17070)}=7.08, p=.008\right)$. The lowest congruence effect on RTs $(€ 0=53 \pm 121$ $\mathrm{ms} ; 1$ cent $=57 \pm 121 \mathrm{~ms} ; € 1=62 \pm 118 \mathrm{~ms}$ ) was observed in the nonrewarded situation (Congruence $\times$ Reward, RT $-€ 1$ vs. $€ 0, F_{(1,16296)}=15.46, p<.0001 ; € 0$ vs. 1 cent, $F_{(1,16316)}=$ $3.00, p=.08)$, while the lowest congruence effect on accuracy $(€ 0=5.4 \% ; 1$ cent $=4.7 \%$; $€ 1$ $=6.4 \%$ ) was found in the low-reward situation (Congruence $\times$ Reward, accuracy $-€ 1$ vs. $€ 0$, $F_{(1,17019)}=0.74, p=.39 ; € 0$ vs. 1 cent, $\left.F_{(1,17001)}=2.82, p=.09\right)$.

Group comparisons (Table 2) showed that the groups differed neither on the overall error rate (Group, $F_{(1,70)}=0.12, p=.73$ ) nor on the overall RT, even if patients with PD tended to be faster (Group, $F_{(1,70)}=3.20, p=.07$ ). The congruence effect on RTs was greater in patients than in HC (62 ms vs. 53 ms) (Group $\times$ Congruence, RT, $\left.F_{(1,24478)}=15.00, p=.0001\right)$, but the congruence effect on accuracy was equivalent in both groups (Group $\times$ Congruence, accuracy, $\left.F_{(1,25545)}=2.30, p=.13\right)$. Irrespective of congruence status, both groups were impacted by the promised rewards, but only in terms of speed (Group $\times$ Reward, RT, $F_{(2,24478)}=518.01, p<$ .0001), not accuracy, (Group $\times$ Reward, accuracy, $\left.F_{(1,25545)}=2.79, p=.25\right)$. The influence of promised rewards on the congruence effect did, however, differ between the groups for RTs $\left(\right.$ Group $\times$ Congruence $\times$ Reward, RT, $\left.F_{(2,24478)}=5.81, p=.05\right)$, though not for errors (Group $\times$ Congruence $\times$ Reward, accuracy, $\left.F_{(2,25545)}=4.08, p=.13\right)($ Fig. 2$)$. The promise of a reward increased the congruence effect in HC (Congruence $\times$ Reward, RT - HC group, $F_{(2,12280)}=$ 22.62, $p=.05$ ) but not in PD patients, for whom no significant interaction was observed (Congruence $\times$ Reward, RT - PD group, $\left.F_{(2,12198)}=1.24, p=.54\right)$. Thus, the congruence effect was modulated by incentive motivation in HC but not in patients with medically treated PD (Fig. 2). 
Table 2: Mean response times (RTs) and error rates for the rewarded Simon task for the HC and PD groups

\begin{tabular}{|c|c|c|c|c|c|}
\hline \multicolumn{2}{|l|}{0 euro } & \multicolumn{2}{|l|}{1 cent } & \multicolumn{2}{|l|}{1 euro } \\
\hline Congruent & Noncongruent & Congruent & Noncongruent & Congruent & Noncongl \\
\hline 509 (14) & $556(11)$ & 492 (14) & $542(12)$ & 469 (12) & $529(12)$ \\
\hline 487 (14) & $545(14)$ & $470(68)$ & $530(16)$ & 455 (15) & 515 (17) \\
\hline
\end{tabular}

Error rate (\%)

$\begin{array}{lllllll}\mathrm{HC} & 1.4(0.4) & 6.4(1.1) & 1.3(0.3) & 6.7(1.2) & 1.0(0.3) & 7.7(1.1) \\ \mathrm{PD} & 1.2(0.4) & 7.1(0.9) & 2.0(0.5) & 6.1(1.0) & 1.5(0.4) & 7.7(1.0)\end{array}$

Note. Values in parentheses are standard errors of the mean (SEMs).

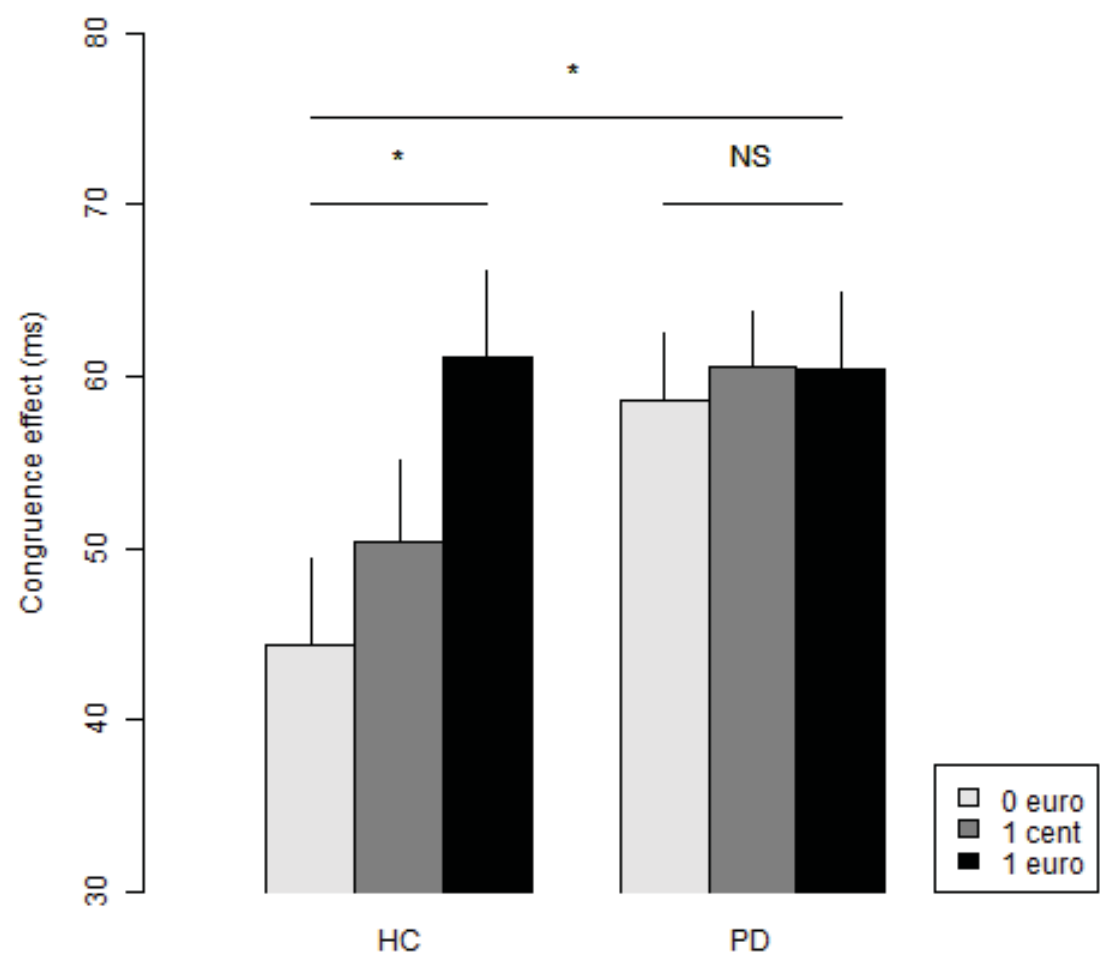

Figure 2: Congruence effect on RTs according to promised rewards in HC and patients with PD. Error bars represent SEMs. HC = Healthy controls; $\mathrm{PD}=$ patients with Parkinson's disease; *p $=.05 ; \mathrm{NS}=$ non-significant 


\subsection{Conditional accuracy function.}

The effects of promised rewards on the automatic route were analyzed by plotting response accuracy as a function of RTs, divided into three bins. First, all participants had greater difficulty producing fast correct responses than slow ones in the noncongruent condition. The significant Bin $\times$ Congruence interaction, $F_{(2,25545)}=247.56, p<.0001$, and the bin-by-bin comparison showed that the congruence effect was dependent upon the speed, as the greatest effect was observed for the fastest responses (Bin $\times$ Congruence, first vs. second bin, $F_{(1,17073)}$ $=112.54, p<.0001$; second vs. third bin: $F_{(2,16874)}=32.97, p<.0001$; first vs. third bin: $F_{(1 \text {, }}$ 17143) $=232.37, p<.0001)$. Second, the promise of a reward had no impact on the congruence effect (Congruence $\times$ Reward, $\left.F_{(2,25545)}=1.50, p=.47\right)$, whatever the RT $($ Bin $\times$ Congruence $\times$ Reward, $\left.F_{(4,25545)}=0.91, p=.92\right)$. These results were confirmed by the analysis of the error rate for the fastest responses (first bin), in which no Congruence $\times$ Reward interaction was observed (Congruence $\times$ Reward, first bin, $F_{(2,8671)}=1.28, p=.53$ ) despite significant main effects of reward (Reward, first bin, $F_{(2,8671)}=9.97, p=.007$ ), and congruence (Congruence, first bin, $\left.F_{(1,8671)}=337.38, p<.0001\right)$. Thus, it was the fastest responses that appeared most prone to capture by the irrelevant dimension of the stimulus (congruence effect on first bin), and the promise of a reward decreased the accuracy of the fastest responses (reward effect on first bin). However, the promised rewards did not increase the risk of impulsive action selection induced by the irrelevant dimension of the stimulus.

Group comparisons (Figs. 3a and 3b) showed that, irrespective of the influence of the reward, the temporal dynamics of the congruence effect on accuracy did not differ between groups (Group $\times$ Congruence $\times$ Bin, $\left.F_{(2,25545)}=4.74, p=.09\right)$. These results were confirmed by the analysis of the error rate for the fastest responses (Group $\times$ Congruence, first bin, $F_{(1,8671)}=$ $1.45, p=.22$ ). The greatest congruence effect was observed for the fastest responses (first bin) 
in both the HC group (Congruence, first bin $-\mathrm{HC}$ group, $F_{(1,4343)}=168.94, p<.0001$ ), and the PD group (Congruence, first bin - PD group, $F_{(1,4328)}=169.45, p<.0001$ ). When we examined the influence of the promised rewards on the temporal dynamics of the automatic route, we observed a significant difference between the groups (Group $\times$ Congruence $\times$ Reward $\times$ Bin, $F_{(4,25545)}=10.78, p=.03$ ) (Figs. 3a and 3b), but this effect was not supported by the fastest responses (Group $\times$ Congruence $\times$ Reward, first bin, $F_{(2,8671)}=4.80, p=.09$ ). The promise of a reward did not influence impulsive action selection in either HC (Congruence $\times$ Reward, first bin - HC group, $F_{(2,4343)}=3.57, p=.17$ ) or patients with PD (Congruence $\times$ Reward, first bin $-\mathrm{PD}$ group, $\left.F_{(2,4328)}=2.51, p=.28\right)($ Fig. 3), despite the presence of similar reward effects in the first bin for both groups (Group $\times$ Reward, first bin, $\left.F_{(2,8671)}=1.68, p=.43\right)$. Rewards decreased the accuracy of the fastest responses (first bin) in both groups, irrespective of the congruence condition (errors rates, first bin - HC group: $€ 0=$ $8.6 \%, 1$ cent $=9.0 \%, € 1=10.7 \%$; PD group: $€ 0=8.5 \%, 1$ cent $=7.4 \%, € 1=9.7 \%$ ). This effect was not restricted to the fastest responses (Reward $\times \operatorname{Bin}, F_{(4,25545)}=2.9, p=.57$ ).

A

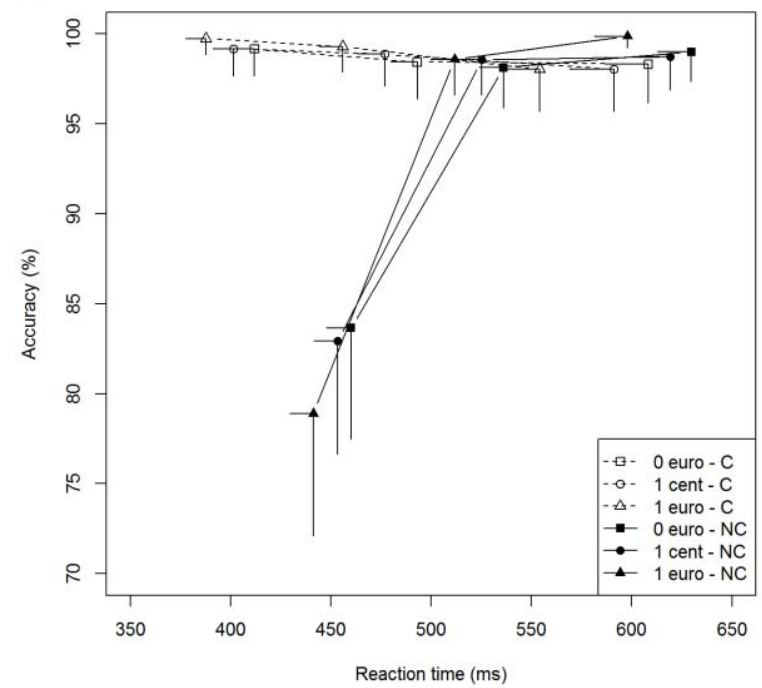

B

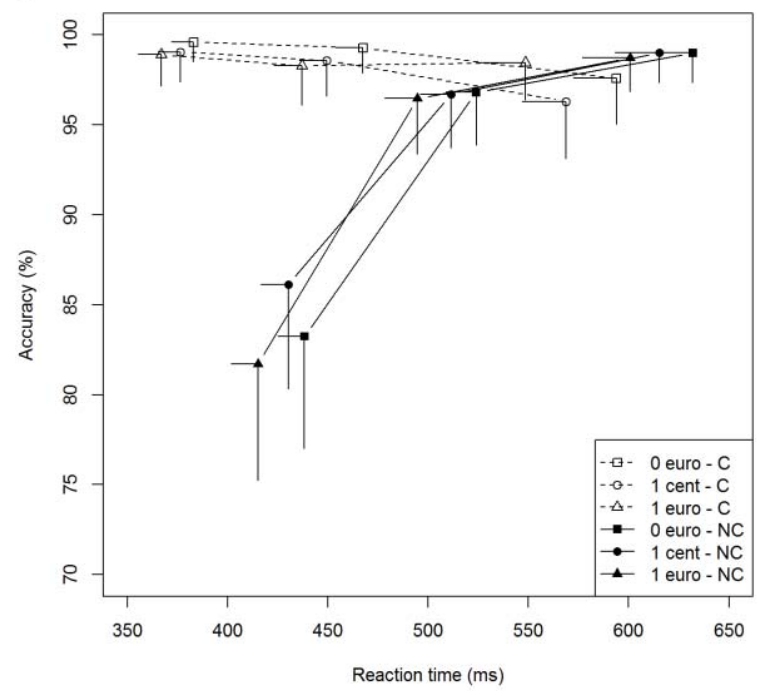

Figure 3: Conditional accuracy function (CAF) for congruent and noncongruent trials according to promised rewards for (A) the HC group and (B) the PD group. Each bin represents the mean score of a third of the overall dataset. Error bars represent SEMs. C = congruent; NC = noncongruent. 


\subsection{Delta plots.}

The effects of promised rewards on the controlled route were analyzed by plotting the congruence effect against RTs, divided into three bins, and by estimating the slopes between the bins (2 slopes, Figs. 4a and 4b).

First, suppression of inappropriate activation was most effective for the slowest responses (Slope, $\left.F_{(1,432)}=4.61, p=.03\right)$. The second slope was the strongest showing that the selective suppression of inappropriate activations was more effective for the slowest responses. Second, promised rewards increased the difficulty of suppressing inappropriate activation (Reward, $F_{(2,432)}=6.50, p=.04$. Compared with the nonrewarded situation, the slopes were significantly weaker for $€ 1$ (Reward, $€ 1$ vs. $€ 0, F_{(1,288)}=6.62, p=.01$ ), and tended toward significance for 1 cent (Reward, 1 cent vs. $€ 0, F_{(1,288)}=3.07, p=.08$ ), whereas there was no significant difference between $€ 1$ and 1 cent (Reward, $€ 1$ vs. 1 cent, $F_{(1,288)}=0.49, p=.48$ ). This effect was not dependent on RT (Reward $\times$ Slope, $F_{(2,432)}=2.64, p=.27$ ).

Group comparisons showed that patients with PD had greater difficulty suppressing and surpassing inappropriate activation (Group, $F_{(1,432)}=6.88, p=.009$ ) and this difference was dependent on RTs (Group $\times$ Slope, $F_{(1,432)}=6.99, p=.008$ ). Patients with PD had the greatest difficulty suppressing inappropriate activation in the fastest responses (Group, first slope: $F_{(1 \text {, }}$ 216) $=10.15, p=.001$; second slope: $\left.F_{(1,216)}=0.67, p=.41\right)$. More specifically, the ability of HC to suppress inappropriate activation was independent of RT (Slope, HC group, $F_{(1,216)}=$ $0.13, p=.71$ ) (first slope, $m=-0.13$; second slope, $m=-0.17$ ), whereas patients with PD needed more time to effectively activate selective suppression (Slope, PD group, $F_{(1,216)}=$ 10.85, $p=.001$ ) (first slope: $m=0.02$; second slope: $m=-0.12$ ).

In addition, the effect of promised rewards on the suppression of inappropriate activation appeared not to differ between groups (Group $\times$ Reward, $F_{(2,432)}=0.79, p=.67$ ), suggesting 
that both groups found it equally difficult to suppress inappropriate activation in rewarded situations. Nor did the effect of promised rewards on the temporal dynamics of the suppression of inappropriate activation differ between groups (Group $\times$ Slope $\times$ Reward, $F_{(2}$, $\left.{ }_{432)}=0.79, p=.67\right)$. However, as the delta plots for the two groups looked very different (Figs. 4a and 4b), we inspected the effects of reward in each group separately. This inspection showed that modulation by promised rewards took place in the PD group (Reward, PD group, $\left.F_{(2,432)}=6.34, p=.04\right)$, but not in the HC group (Reward, HC group, $\left.F_{(2,432)}=1.19, p=.55\right)$. Selective response suppression was modulated by the incentive motivation condition in the PD group (€1: $m=-0.01 ; 1$ cent: $m=-0.03 ; € 0: m=-0.10$ ), whereas the efficiency of this process did not vary in HC (€1: $m=-0.11 ; 1$ cent: $m=-0.15 ; € 0: m=-0.18)$.

A

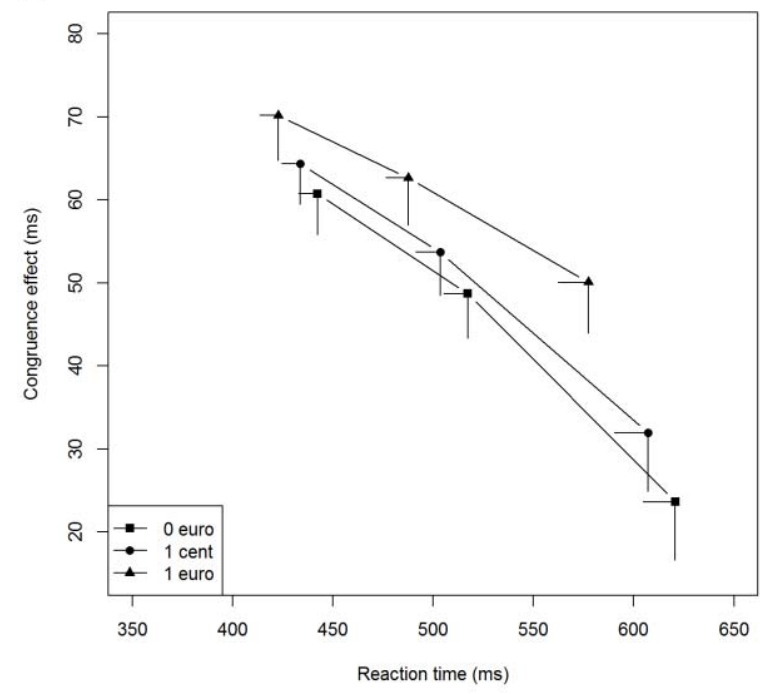

B

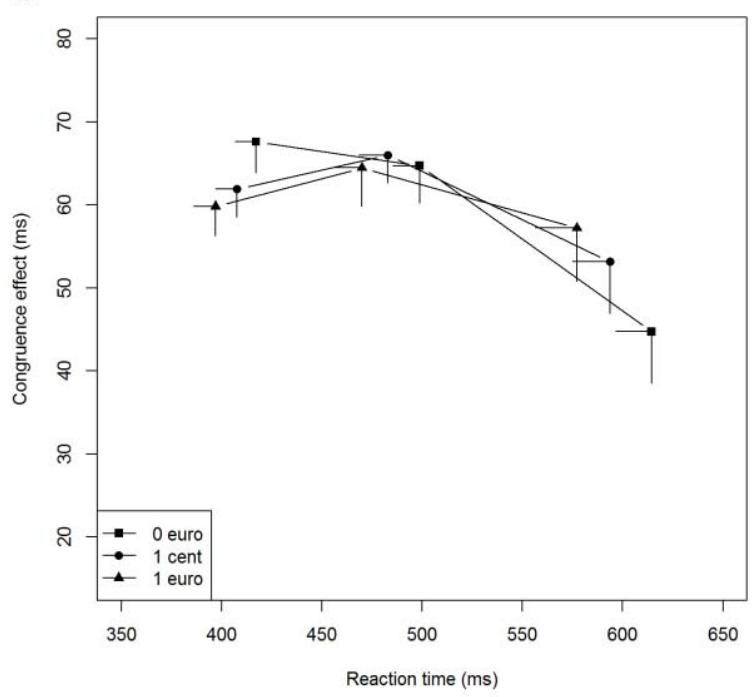

Figure 4: Delta plots of the congruence effect (noncongruent minus congruent RTs for correct responses) according to promised rewards (A) in HC and (B) in patients with PD. Each bin represents the mean score of a third of the overall dataset. The position of each bin corresponds to the mean RT on the $x$-axis and the mean congruence effect on RTs on the $y$-axis. Error bars represent SEMs. 


\section{Discussion}

The current study was designed to clarify the influence of promised rewards on the temporal aspects of information processing in conflict situations, in both $\mathrm{HC}$ and patients with medically treated PD. To this end, we administered a rewarded Simon task to 36 HC and 36 patients with PD. First, we analyzed the results according to the dual-route model, using the classic congruence effect. The strength of the congruence effect tells us about the amount of cognitive effort needed to suppress and overcome inappropriate activation [8]. When we compared the congruence effects in different reward situations, we found that the promise of a reward increased the cognitive effort needed to resolve conflict, but only in HC. Patients with medically treated PD did not modulate their cognitive effort according to incentive motivation. Second, we carried out analyses based on the activation-suppression model [1113]. This model allowed us to investigate the temporal dynamics of both impulse selection and impulse suppression. We found that although the promise of a reward did not influence impulsive action selection in either group, it did make selective impulse suppression harder for patients with PD.

As expected, both groups had more difficulty producing correct responses in noncongruent situations than in congruent ones, as observed with the classic congruence effect measured on both accuracy and RTs. A previous study had found that the promise of a reward increases the speed of responses in relation to the size of the reward, irrespective of congruence status [20]. This suggested that promised rewards increase time pressure. In our study, however, promised rewards only increased the congruence effect in HC. The promise of a reward therefore increased time pressure for the HC group, and this pressure made conflict resolution harder. Our finding that promised rewards worsen the congruence effect contrasts with studies reporting either no influence of promised rewards, as measured with behavioral data [18,21], 
or a positive influence [20]. The lack of a significant interaction in the first two studies may have been due to insufficient statistical power, whereas methodological variants, notably concerning the threshold set to obtain the reward, provide a better explanation for the difference with Padmala and Pessoa (2011)'s study. In our study, the full reward was delivered when the speed of the correct response was one sixth below the mean baseline RT. In the study by Padmala and Pessoa (2011), however, the threshold RT for a full reward was actually longer than the mean baseline RT [20]. Thus, a full reward was granted for only the fastest correct responses in our study, but for all correct responses except the very slowest ones in the study by Padmala and Pessoa (2011). We hypothesize that feedback (in the present case, a monetary reward) provides additional information about task difficulty and leads to different cognitive strategies. Our results suggest that the promise of a reward only induces time pressure when rewards are restricted to the fastest responses, which in turn makes conflict resolution harder. This assumption fits with the results of conflict tasks completed under time pressure [30,31]. It could also fit with the choking under pressure model that postulates that our performances decrease when, under excessive pressure of incentive motivation, we try but fail to do our best [32-34]. In other words, the presence of expected rewards can trigger a state of excessive arousal, resulting in impaired performance in speedstress situations.

Although the promise of a reward increased time pressure, this stress did not make conflict resolution any harder for patients with medically treated PD, in contrast to HC. This is in accordance with a recent study that also used a modified Simon task and showed that, in PD patients, neither rewarding nor punishing modulates conflict resolution, as measured by the congruence effect [19]. As the patients in both this study and ours were under medication during the task and had only mild disease severity (as shown by the UPDRS-III scores off 
medication: 22.1 vs. $28.3 \pm 9.7$ in the present study), we can hypothesize that dopamine treatment was responsible for these effects. Effectively, dopamine medication may have overdosed the limbic ventral dopamine system, relatively preserved in mild affected patients in contrast to the dorsal motor system. This could in turn impair reward processing, preventing conflict resolution from being modulated by motivation level. If this hypothesis is correct, patients with medication no longer modulate the cognitive effort needed to resolve conflict situations according to the magnitude of the incentive $[19,35]$, whereas in patients without medication, promised rewards still allow for the modulation of cognitive control [36]. Our findings could be explained by PD disease progression creating an imbalance between relatively intact dopamine levels in the ventral striatum and severely depleted dopamine levels in the dorsal striatum, possibly leading to increased reward-related impulsivity in PD patients off medication. One alternative hypothesis is that conflict resolution was so impaired in our patients with PD that incentive motivation could no longer modulate it. This would fit with our results showing that the congruence effect was greater in patients than in HC.

Complementary data analyses based on the activation-suppression model yielded additional information about dynamic impulse selection and suppression [11-13]. In this model, the fastest actions are the most impulsive, as they are driven by the irrelevant stimulus information, leading to more erroneous responses. Accordingly, we observed higher error rates for the fastest responses in noncongruent situations in both groups. Furthermore, in highreward situations, the error rate increased for the fastest responses. As incentives increase response speed, we would expect promised rewards to increase impulse selection which, in turn, would result in increased error rates in noncongruent situations. It has been shown that when the instructions given to participants emphasize speed rather than accuracy in a Simon 
task, time pressure favors impulsive action selection [30,37]. However, we did not find any significant modulation of impulsive action selection by promised rewards.

We failed to observe any difference between the two groups concerning impulsive action selection, irrespective of both congruence and reward. Patients and HC did not differ in terms of impulsive response selection. The lack of a group difference was not surprising, as previous studies had failed to demonstrate pathological response capture by irrelevant stimulus information in a similar population of patients with PD to ours, that is, with mild disease severity. Large response capture was only observed in a subgroup of patients with more severe PD [31,38].

According to the activation-suppression model, the slowest actions are the least influenced by inappropriate activation driven by irrelevant stimulus information, as there is enough time for the selective inhibition of the irrelevant response tendency to get going. Thus, as our delta plots showed, the congruence effect, as measured with correct response RTs, was weaker for the slower responses.

The group comparison showed that the patients with PD needed more time to efficiently suppress inappropriate activation (first slope). However, when selective suppression became effective (second slope), there ceased to be a difference in suppression efficiency between the two groups. This lack of a significant difference in the second part of the distribution contrasts with previous studies showing that patients with PD have impaired impulse suppression even when they respond slowly enough to allow the suppression process to get going [30,31,3741]. This difference can be explained by the fact that we estimated the temporal dynamics of the responses with only three bins, compared with between five and seven bins in the abovecited studies, leading to a less accurate representation of response distribution. Although our task contained no fewer than 360 trials, in order for there to be a satisfactory number (20) of 
trials in each bin, we had to limit the number of bins in the analysis to compensate for the introduction of different reward conditions [42]. Adding more trials per condition could have been deleterious, especially for the patients with PD, owing to their attention deficit. In addition, promised rewards only modulated the ability to suppress impulsive responses in the PD group. The slopes of the delta plots were more negative in the nonrewarded situation than in the rewarded one, indicating that promised rewards made selective suppression harder for the patients with PD. Furthermore, the analysis based on the activation-suppression model showed that promised rewards modulated the efficiency of a specific aspect of cognitive action control in patients, namely selective impulse suppression. The results of the present study fit with previous studies reporting less efficient cognitive inhibition in patients with PD when they are instructed to favor response speed over accuracy [30]. Promised rewards appeared to induce time pressure which, in turn, made the selective suppression of impulse activation harder for patients with medically treated PD. By contrast, in HC, promised rewards did not modulate selective impulse suppression, suggesting that inhibitory control was still efficient, irrespective of the speed pressure induced by incentive context.

In conclusion, we found that promised rewards had a deleterious effect on cognitive action control in both HC and patients with medically treated PD. However, this effect differed between the two groups. The cognitive cost induced by conflict situations in the rewarded context could still be observed in HC but not in patients. Our results suggest that patients with Parkinson's disease have difficulties to apply an efficient cognitive action control and cannot modulate the related cognitive abilities according to the incentive motivation context. We may thus formulate two hypotheses, not mutually exclusive. First, the lack of modulation in cognitive action control according to the incentive context would directly result from the impairment of the system supporting the cognitive action control. Second, the dopaminergic 
overdose of the limbic ventral system, relatively preserved in mild Parkinson's disease, would impair the neural coding of the incentive information. Additionally, patients had greater difficulty suppressing inappropriate activation in rewarded situations. This indicates that incentive motivation can modulate at least one cognitive process involved in cognitive action control in patients with medically treated PD. Creating experimental tasks with many repetitions of incentive situations without losing the incentive effect will be a challenge for future studies. More trials, and thus more bins per participant and per condition, would refine the modeling of how incentive motivation influences impulse selection and suppression in both $\mathrm{HC}$ and PD. As it is the case for most experimental tasks, implication of our results on activities of daily living remains speculative since such experimental tasks have rather low ecological validity. In order to better evaluate how the experimental impairment that we observed may impact the current clinical practice, it would be of interest to complete such studies with appropriate methods for the ecological assessment of executive functioning $[43,44]$. Moreover, complementary studies comparing patients on versus off medication are essential if we are to improve our understanding of how dopamine treatment influences the modulation of both impulse activation and suppression according to the incentive context. Finally, it would be interesting to investigate the relationship between cognitive action control abilities in an incentive context and the medication-induced impulse control disorders observed in some patients with PD.

\section{Acknowledgments}

We would like to thank Elizabeth Wiles-Portier for revising the English style. This study was supported by the University of Rennes 1 . 


\section{References}

[1] A. Diamond, Executive Functions, Annu. Rev. Psychol. 64 (2013) 135-168. doi:10.1146/annurev-psych-113011-143750.

[2] A. Kudlicka, L. Clare, J.V. Hindle, Executive functions in Parkinson's disease: Systematic review and meta-analysis, Mov. Disord. 26 (2011) 2305-2315. doi:10.1002/mds.23868.

[3] J.R. Stroop, Studies of interference in serial verbal reactions., J. Exp. Psychol. 18 (1935) 643.

[4] B.A. Eriksen, C.W. Eriksen, Effects of noise letters upon the identification of a target letter in a nonsearch task, Percept. Psychophys. 16 (1974) 143-149.

[5] J.R. Simon, The effects of an irrelevant directional cue on human information processing., in: Stimulus-response Compat. Integr. Perspect., R. W. Proctor \& T. G. Reeve, 1990: pp. 31-86.

[6] R. De Jong, C.C. Liang, E. Lauber, Conditional and unconditional automaticity: a dualprocess model of effects of spatial stimulus-response correspondence, J. Exp. Psychol. Hum. Percept. Perform. 20 (1994) 731-750.

[7] M. Eimer, B. Hommel, W. Prinz, SR compatibility and response selection, Acta Psychol. (Amst.). 90 (1995) 301-313.

[8] S. Kornblum, T. Hasbroucq, A. Osman, Dimensional overlap: cognitive basis for stimulus-response compatibility--a model and taxonomy, Psychol. Rev. 97 (1990) 253270.

[9] Inhibition in cognition, American Psychological Association, Washington, DC, US, 2007.

[10] K.R. Ridderinkhof, B.U. Forstmann, S.A. Wylie, B. Burle, W.P.M. van den Wildenberg, Neurocognitive mechanisms of action control: resisting the call of the Sirens, Wiley Interdiscip. Rev. Cogn. Sci. 2 (2011) 174-192. doi:10.1002/wcs.99.

[11] K.R. Ridderinkhof, Activation and suppression in conflict tasks: Empirical clarification through distributional analyses., in: Common Mech. Percept. Action Atten. Perform. Ser., Oxford: Oxford University Press, 2002: pp. 494-519. http://dare.uva.nl/record/1/419995 (accessed November 10, 2015).

[12] K.R. Ridderinkhof, Micro- and macro-adjustments of task set: activation and suppression in conflict tasks, Psychol. Res. 66 (2002) 312-323. doi:10.1007/s00426-0020104-7.

[13] K.R. Ridderinkhof, W.P.M. van den Wildenberg, J. Wijnen, B. Burle, Response inhibition in conflict tasks is revealed in delta plots, in: Cogn. Neurosci. Atten., n.d.

[14] M. Eimer, Facilitatory and inhibitory effects of masked prime stimuli on motor activation and behavioural performance, Acta Psychol. (Amst.). 101 (1999) 293-313.

[15] M. Eimer, F. Schlaghecken, Effects of masked stimuli on motor activation: behavioral and electrophysiological evidence, J. Exp. Psychol. Hum. Percept. Perform. 24 (1998) 1737-1747.

[16] W.P.M. van den Wildenberg, S.A. Wylie, B.U. Forstmann, B. Burle, T. Hasbroucq, K.R. Ridderinkhof, To head or to heed? Beyond the surface of selective action inhibition: a review, Front. Hum. Neurosci. 4 (2010) 222. doi:10.3389/fnhum.2010.00222.

[17] K.C. Berridge, Motivation concepts in behavioral neuroscience, Physiol. Behav. 81 (2004) 179-209. doi:10.1016/j.physbeh.2004.02.004.

[18] E. Aarts, D.L. Wallace, L.C. Dang, W.J. Jagust, R. Cools, M. D’Esposito, Dopamine and the cognitive downside of a promised bonus, Psychol. Sci. 25 (2014) 1003-1009. doi:10.1177/0956797613517240. 
[19] E. Di Rosa, S. Schiff, F. Cagnolati, D. Mapelli, Motivation-cognition interaction: how feedback processing changes in healthy ageing and in Parkinson's disease, Aging Clin. Exp. Res. (2015). doi:10.1007/s40520-015-0358-8.

[20] S. Padmala, L. Pessoa, Reward reduces conflict by enhancing attentional control and biasing visual cortical processing, J. Cogn. Neurosci. 23 (2011) 3419-3432. doi:10.1162/jocn_a_00011.

[21] B. van den Berg, R.M. Krebs, M.M. Lorist, M.G. Woldorff, Utilization of reward-prospect enhances preparatory attention and reduces stimulus conflict, Cogn. Affect. Behav. Neurosci. 14 (2014) 561-577. doi:10.3758/s13415-014-0281-z.

[22] A.J. Hughes, Y. Ben-Shlomo, S.E. Daniel, A.J. Lees, What features improve the accuracy of clinical diagnosis in Parkinson's disease: a clinicopathologic study, Neurology. 42 (1992) 1142-1146.

[23] S. Mattis, Dementia rating scale professional manual, 1988.

[24] D.V. Sheehan, Y. Lecrubier, K.H. Sheehan, P. Amorim, J. Janavs, E. Weiller, et al., The Mini-International Neuropsychiatric Interview (M.I.N.I.): the development and validation of a structured diagnostic psychiatric interview for DSM-IV and ICD-10, J. Clin. Psychiatry. 59 Suppl 20 (1998) 22-33;quiz 34-57.

[25] S.A. Montgomery, M. Asberg, A new depression scale designed to be sensitive to change, Br. J. Psychiatry J. Ment. Sci. 134 (1979) 382-389.

[26] M.M. Hoehn, M.D. Yahr, Parkinsonism: onset, progression and mortality, Neurology. 17 (1967) 427-442.

[27] R.S. Schwab, A.C. England, E. Peterson, Akinesia in Parkinson's disease, Neurology. 9 (1959) 65-72.

[28] J. Pinheiro, D. Bates, S. DebRoy, D. Sarkar, EISPACK authors, R-core, nlme: Linear and Nonlinear Mixed Effects Models, 2015. https://cran.rproject.org/web/packages/nlme/index.html (accessed September 27, 2015).

[29] D. Bates, M. Maechler, B. Bolker, S. Walker, R.H.B. Christensen, H. Singmann, et al., Ime4: Linear mixed-effects models using "Eigen" and S4, 2015. https://cran.rproject.org/web/packages/Ime4/index.html (accessed September 27, 2015).

[30] N.C. van Wouwe, W.P.M. van den Wildenberg, D.O. Claassen, K. Kanoff, T.R. Bashore, S.A. Wylie, Speed pressure in conflict situations impedes inhibitory action control in Parkinson's disease, Biol. Psychol. 101 (2014) 44-60. doi:10.1016/j.biopsycho.2014.07.002.

[31] S.A. Wylie, W.P.M. van den Wildenberg, K.R. Ridderinkhof, T.R. Bashore, V.D. Powell, C.A. Manning, et al., The effect of Parkinson's disease on interference control during action selection, Neuropsychologia. 47 (2009) 145-157. doi:10.1016/j.neuropsychologia.2008.08.001.

[32] R.F. Baumeister, Choking under pressure: self-consciousness and paradoxical effects of incentives on skillful performance, J. Pers. Soc. Psychol. 46 (1984) 610-620.

[33] S.L. Beilock, C.A. Kulp, L.E. Holt, T.H. Carr, More on the fragility of performance: choking under pressure in mathematical problem solving, J. Exp. Psychol. Gen. 133 (2004) 584600. doi:10.1037/0096-3445.133.4.584.

[34] D. Mobbs, D. Hassabis, B. Seymour, J.L. Marchant, N. Weiskopf, R.J. Dolan, et al., Choking on the Money Reward-Based Performance Decrements Are Associated With Midbrain Activity, Psychol. Sci. 20 (2009) 955-962. doi:10.1111/j.14679280.2009.02399.x. 
[35] D. Mapelli, E. Di Rosa, M. Cavalletti, S. Schiff, S. Tamburin, Decision and dopaminergic system: an ERPs study of lowa gambling task in Parkinson's disease, Front. Psychol. 5 (2014) 684. doi:10.3389/fpsyg.2014.00684.

[36] E. Aarts, R.C. Helmich, M.J.R. Janssen, W.J.G. Oyen, B.R. Bloem, R. Cools, Aberrant reward processing in Parkinson's disease is associated with dopamine cell loss, Neurolmage. 59 (2012) 3339-3346. doi:10.1016/j.neuroimage.2011.11.073.

[37] S.A. Wylie, W.P.M. van den Wildenberg, K.R. Ridderinkhof, T.R. Bashore, V.D. Powell, C.A. Manning, et al., The effect of speed-accuracy strategy on response interference control in Parkinson's disease, Neuropsychologia. 47 (2009) 1844-1853. doi:10.1016/j.neuropsychologia.2009.02.025.

[38] S.A. Wylie, K.R. Ridderinkhof, T.R. Bashore, W.P.M. van den Wildenberg, The effect of Parkinson's disease on the dynamics of on-line and proactive cognitive control during action selection, J. Cogn. Neurosci. 22 (2010) 2058-2073. doi:10.1162/jocn.2009.21326.

[39] D.O. Claassen, W.P.M. van den Wildenberg, M.B. Harrison, N.C. van Wouwe, K. Kanoff, J.S. Neimat, et al., Proficient motor impulse control in Parkinson disease patients with impulsive and compulsive behaviors, Pharmacol. Biochem. Behav. 129 (2015) 19-25. doi:10.1016/j.pbb.2014.11.017.

[40] S.A. Wylie, D.O. Claassen, H.M. Huizenga, K.D. Schewel, K.R. Ridderinkhof, T.R. Bashore, et al., Dopamine agonists and the suppression of impulsive motor actions in Parkinson disease, J. Cogn. Neurosci. 24 (2012) 1709-1724. doi:10.1162/jocn_a_00241.

[41] S.A. Wylie, W. van den Wildenberg, K.R. Ridderinkhof, D.O. Claassen, G.F. Wooten, C.A. Manning, Differential susceptibility to motor impulsivity among functional subtypes of Parkinson's disease, J. Neurol. Neurosurg. Psychiatry. 83 (2012) 1149-1154. doi:10.1136/jnnp-2012-303056.

[42] S.A. Wylie, K.R. Ridderinkhof, W.J. Elias, R.C. Frysinger, T.R. Bashore, K.E. Downs, et al., Subthalamic nucleus stimulation influences expression and suppression of impulsive behaviour in Parkinson's disease, Brain J. Neurol. 133 (2010) 3611-3624. doi:10.1093/brain/awq239.

[43] P.W. Burgess, N. Alderman, C. Forbes, A. Costello, L.M.-A. Coates, D.R. Dawson, et al., The case for the development and use of "ecologically valid" measures of executive function in experimental and clinical neuropsychology, J. Int. Neuropsychol. Soc. JINS. 12 (2006) 194-209. doi:10.1017/S1355617706060310.

[44] N. Chaytor, M. Schmitter-Edgecombe, The ecological validity of neuropsychological tests: a review of the literature on everyday cognitive skills, Neuropsychol. Rev. 13 (2003) 181-197. 


\section{Highlights}

Action control in incentive context in PD 